\title{
Second International Consensus Conference on Advanced Breast Cancer (ABC2), Lisbon, 11/09/2013: The German Perspective
}

\author{
Nadia Harbeck ${ }^{\mathrm{a}}$ Norbert Marschner ${ }^{\mathrm{b}}$ Michael Untch ${ }^{c}$ Thomas Decker ${ }^{\mathrm{d}}$ \\ Susanna Hegewisch-Becker ${ }^{\mathrm{C}}$ Christian Jackisch ${ }^{f}$ Jens Huober ${ }^{g}$ \\ Hans-Joachim Lück ${ }^{h}$ Gunter von Minckwitz ${ }^{i}$ Anton Scharl ${ }^{k}$ Andreas Schneeweiss \\ Hans Tesch $^{m}$ Anja Welt ${ }^{n} \quad$ Rachel Wuerstlein $^{\circ} \quad$ Christoph Thomssen $^{p}$
}

${ }^{a}$ Brustzentrum, Frauenklinik der Universität München; ' ${ }^{b}$ Gemeinschaftspraxis für interdisziplinäre Onkologie und Hämatologie, Freiburg; 'Klinik für Gynäkologie, HELIOS Klinikum Berlin Buch; 'Gemeinschaftspraxis Onkologie Ravensburg; ${ }^{\circ}$ Onkologische Schwerpunktpraxis

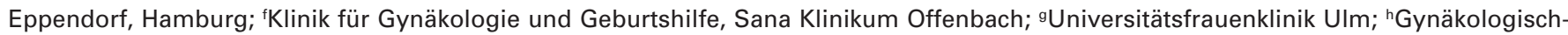
Onkologische Praxis Hannover; i GBG Forschungs GmbH Neu-Isenburg, Universitäts-Frauenklinik Frankfurt; ${ }^{k}$ Frauenklinikum des Klinikums St. Marien, Amberg; 'Sektion Gynäkologische Onkologie, Nationales Zentrum für Tumorerkrankungen, Heidelberg; ${ }^{\mathrm{m} O n k o l o g i s c h e}$ Gemeinschaftspraxis Frankfurt; "Innere Klinik (Tumorforschung), Westdeutsches Tumorzentrum, Universitätsklinikum Essen; ${ }^{\circ}$ Brustzentrum, Frauenklinik, Klinikum der Universität München; PUniversitätsklinik und Poliklinik für Gynäkologie, Martin-Luther-Universität, Halle (Saale), Deutschland

\section{Keywords}

Locally advanced breast cancer - LABC .

Metastatic breast cancer - MBC - Consensus · AGO

\section{Summary}

The Advanced Breast Cancer Second International Consensus Conference $(A B C 2)$ on diagnosis and treatment of advanced breast cancer took place in Lisbon, Portugal, on November 7-9, 2013. The focus of the conference was inoperable, locally advanced breast cancer. The diagnosis and treatment of metastatic breast cancer had already been discussed 2 years before at the ABC 1 Consensus and were only updated regarding special issues as part of this year's ABC2 Consensus. Like 2 years ago, a working group of German breast cancer experts commented on the voting results of the $A B C$ panelists, with special consideration of the German guidelines for the diagnosis and treatment of breast cancer (German Gynecological Oncology Working Group (AGO) recommendations, S3 Guideline) in order to adapt them for daily clinical practice in Germany. The goal of both the $A B C$ Consensus and the German comments is to facilitate evidence-based therapy decisions.

Writing committee: Nadia Harbeck, Norbert Marschner, Michael Untch, Christoph Thomssen.

ABC Panel Members: Nadia Harbeck, Christoph Thomssen.

\section{Introduction}

The organizer of the Advanced and Metastatic Breast Cancer Consensus Conferences (ABC) is the 'European School of Oncology' (ESO). The recommendations of this year's Second $\mathrm{ABC}$ Consensus (ABC2) focused on locally advanced breast cancer (LABC) and will be published in coordination with various international specialist associations, the European Society of Medical Oncology (ESMO), the European Society of Mastology (EUSOMA), the Federación Latinoamericana de Mastologia (FLAM), and the Senologic International Society (SIS) in the journal The Breast at the beginning of 2014.

The goal of the ABC Consensus is the international harmonization and standardization of treatment for patients with locally advanced and/or metastatic breast cancer. The topic of this year's ABC2 Consensus was inoperable LABC without distant metastases. Recommendations for the diagnosis and treatment of metastatic breast cancer (stage IV) had already been developed as part of the $\mathrm{ABC} 1$ Consensus Conference in Lisbon in November 2011. Special issues concerning the metastatic setting were updated.

The ABC2 panel consisted of 41 experts from 20 countries, including 2 representatives from Germany (Nadia Harbeck, Christoph Thomssen, table 1). Since every therapy decision must consider individual criteria (e.g., patient preference, comorbidities, pretreatment, tumor biology, burden of disease) in addition to evidence-based data, the ABC panelists deliberately do not recommend individual drugs or therapeutic

\section{KARGER}

Fax +497614520714

Information@Karger.com

www.karger.com
(C) 2014 S. Karger GmbH, Freiburg

1661-3791/14/0091-0052\$39.50/0

Accessible online at:

www.karger.com/brc
Prof. Dr. med. Nadia Harbeck

Brustzentrum der Universität München

Maistrasse-Innenstadt und Großhadern

Marchioninistr. 15, 81377 München, Germany

Nadia.Harbeck@med.uni-muenchen.de 
Table 1: ABC2 Consensus Panelists

\begin{tabular}{ll}
\hline Matti S. Aapro, CH & Clifford A. Hudis, US \\
Fabrice André, FR & Bella Kaufman, IL \\
Carlos H. Barrios, BR & Ian E. Krop, US \\
Jonas Bergh, SE & Nancy U. Lin, US \\
Elizabeth Bergsten Nordström, IT & Musa Mayer, US \\
Laura Biganzoli, IT & Sofia D. Merjaver, US \\
Kimberley L. Blackwell, US & Larry Norton, US \\
Fatima Cardoso, PT & Olivia Pagani, CH \\
Maria João Cardoso, PT & Ann H. Partridge, US \\
Alberto Costa, CH/IT & Frédérique Penault-Llorca, FR \\
Tanja Cufer, SL & Martine J. Piccart, BE \\
Nagi S. El Saghir, LB & Hope S. Rugo, US \\
Lesley Fallowfield, UK & Elzbieta Senkus-Konefka, PL \\
Doris Fenech, MT & George W. Sledge, US \\
Prudence A. Francis, AU & Christoph Thomssen, DE \\
Karen Gelmon, CA & Laura van't Veer, US \\
Sharon H. Giordano, US & Conny Vrieling, CH \\
Joseph Gligorov, FR & Nicola West, UK \\
Nadia Harbeck, DE & Eric P. Winer, US \\
Nehmat Houssami, AU & Binghe Xu, CN \\
\hline
\end{tabular}

sequences. Rather, the goal is to establish a basis for individual, evidence-based therapy decisions. The statements submitted to a recommendation were assessed by the panelists with 'yes' (approval), 'no' (rejection) or 'abstention'. Since the voting results are based on the opinions of experts from different specialties who come from countries with different healthcare systems and resources, it seems reasonable to discuss the voting results from the German perspective in order to adapt them for daily clinical practice in Germany.

\section{Inoperable LABC}

The first part of the $\mathrm{ABC} 2$ meeting focused on the diagnosis and treatment of inoperable LABC. The German experts point out that patients with inoperable LABC may still have - in the absence of distant metastases - a chance of cure; therefore, standard therapies for the treatment of operable primary breast cancer must be considered in this situation. Prior to starting therapy, every patient should be discussed in a multidisciplinary tumor board.

\section{General Aspects of Diagnosis}

There was a high level of agreement among the ABC2 panelists in the general statements on the diagnosis of inoperable LABC: As the basis for an adequate therapy decision, the $\mathrm{ABC} 2$ panelists, in agreement with the German experts, recommend performing a core needle or a punch biopsy before start of therapy in order to determine the histology and biomarker expression of the breast cancer (level of evidence (LoE): IB).

Patients with $\mathrm{LABC}$ have a high risk of distant metastasis. Therefore, the $\mathrm{ABC} 2$ panelists recommend initiating a complete imaging staging, in addition to the medical history, phys- ical examination, and measurement of laboratory values, including chest, abdomen, and bones, prior to starting systemic therapy (LoE: IB). If computed tomography (CT) and/or positron emission tomography (PET)-CT are available, they may be used as part of the staging, according to the $\mathrm{ABC} 2$ recommendation (LoE: IIB).

The German experts basically agree but emphasize that the medical history and physical and laboratory examinations be performed first before any imaging and that conventional procedures - chest X-ray, abdominal ultrasound, bone scan have priority. The German experts recommend a CT scan of the chest primarily for a more precise assessment of locoregional tumor spread. They also emphasized that CT examinations of chest and abdomen have a higher sensitivity for these high-risk patients. The use of newer imaging procedures was discussed. The German experts agreed that new imaging procedures, such as PET-CT or whole-body magnetic resonance imaging (WB-MRI), may be more sensitive in individual cases. Yet, there is currently no evidence that their routine use improves patient prognosis.

\section{General Aspects of Therapy}

The ABC2 panelists and the German experts agreed that patients with primary inoperable LABC initially require systemic treatment before surgery and locoregional radiotherapy. It was also agreed that the vast majority of patients require multimodal treatment, which consists of systemic therapy, surgery, and locoregional radiation (LoE: IA). If the tumor remains inoperable after systemic treatment plus radiation, palliative mastectomy is discouraged. This does not apply if the surgical intervention is assumed to improve the patient's quality of life. The use of autologous tissue flaps for covering resection-related tissue defects should also be considered as an option.

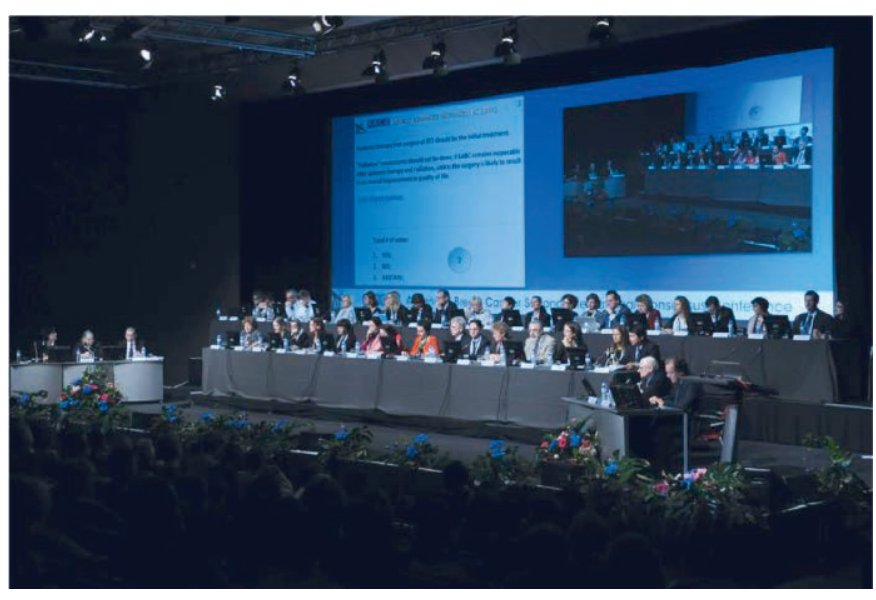

Fig. 1. Consensus Panelists in Lisboa on Saturday morning (November 9, 2013). 
All ABC2 panelists agree that it is possible and sensible for many patients who responded well to neoadjuvant systemic treatment with or without preoperative radiation to undergo subsequent surgery. These patients generally receive a mastectomy plus axillary dissection. Breast-preserving surgery is an option in individual cases. The German experts agree with this statement.

\section{Inflammatory $L A B C$}

The same treatment principles apply to inflammatory LABC and non-inflammatory LABC (LoE: IB). The German experts extend this statement of the $\mathrm{ABC} 2$ panelists by noting that neoadjuvant (primary) chemotherapy (NACT) is a mandatory standard for inflammatory breast cancer.

According to the $\mathrm{ABC} 2$ recommendation, patients with inflammatory LABC should undergo a modified radical mastectomy. This also applies if they respond well to prior NACT (LoE: IB). It has to be noted that the German guidelines even allow breast conservation in selected patients with an excellent response (pathologically confirmed complete remission (pCR)) to NACT and a limited involvement of skin at first presentation (www.ago-online.com). The German experts agree with the $\mathrm{ABC} 2$ recommendation for patients with clinically detectable involvement of lymph nodes and note that axillary treatment for these patients must be based on standards of NACT. Particularly in inflammatory breast cancer, axillary dissection remains the standard and the modified American College of Surgeons Oncology Group (ACOSOG) approach should not be applied.

The ABC2 panelists and German experts agree that patients with inflammatory LABC require locoregional radiotherapy following mastectomy (LoE: IB). Therefore, the $\mathrm{ABC} 2$ panelists do not recommend immediate breast reconstruction following mastectomy (LoE: expert opinion). The German experts recommend reconstruction to repair extensive tissue defects after $\mathrm{R} 0$ resection.

\section{Therapy Decision Depending on the Tumor Biology of $L A B C$}

\section{Triple-Negative $L A B C$}

With a clear majority, the $\mathrm{ABC} 2$ panelists recommend anthracycline- and taxane-based chemotherapy as initial treatment for patients with triple-negative inoperable LABC (estrogen receptor-negative (ER-), progesterone receptor-negative (PR-), human epidermal growth factor receptor 2-negative (HER2-) LABC) (LoE: IA). The German experts agree and add that the established standard anthracycline- and taxane-containing regimens are recommended for triple-negative LABC. If a BRCA mutation is detected, the additional use of a platinum-containing regimen can be considered.

\section{BRCA-Mutated Metastatic Breast Cancer}

In a separate recommendation on BRCA-mutated metastatic breast cancer, the $\mathrm{ABC} 2$ panelists supported the use of platinum-based therapy outside of clinical trials for patients with either endocrine-resistant or triple-negative cancer if they have already been pretreated with anthracyclines and taxanes (adjuvant or metastatic) (LoE: IC). The ABC2 panelists note that poly(ADP-ribose) polymerase (PARP) inhibitors have been very effective in clinical trials in cases of BRCA-mutated breast cancer. But regardless of this, the $\mathrm{ABC} 2$ panelists state that the therapy recommendations for sporadically metastatic breast cancer currently apply for BRCA-mutated metastatic breast cancer. The German experts agree and recommend participation in the upcoming clinical trials with PARP inhibitors.

\section{HER2-Positive LABC}

The majority of the ABC2 panelists recommend concomitant treatment with taxanes and an anti-HER2 therapy for the treatment of patients with HER2-positive inoperable LABC, since concomitant administration is associated with a higher rate of pCRs (LoE: IA). The German experts agree and note that this is consistent with the German guidelines (www.awmf.org, www.ago-online.de).

If the patient also receives an anthracycline, this should be integrated sequentially in the regimen (LoE: IA). If an antiHER2 drug and anthracyclines are administered concomitantly, the pCR rate is not increased, but the risk of cardiac adverse events is higher (LoE: IB).

The German experts recommend to generally use anthracyclines (in combination with cyclophosphamide) followed by taxane and trastuzumab-containing combination therapy - as a rule. The German experts explain this approach with the potentially increased risk of cardiac toxicities under concomitant administration of trastuzumab with the anthracycline, although in the neoadjuvant clinical trials with trastuzumab given concomitantly with the anthracycline (e.g. ACOSOG Z1041, Geparquattro, NOAH) a clinically relevant increase of cardiotoxicity has so far not been observed. Nevertheless, it could not be excluded if used widely in clinical routine. Thus, in the absence of a higher pCR rate, this concomitant schedule is not justified from the German perspective (ACOSOG Z1041 study) [1]. The German experts point out that a substantial increase in the pCR rate can be achieved by dual HER2 blockade with trastuzumab plus pertuzumab or trastuzumab plus lapatinib.

\section{ER-Positive LABC}

For patients with hormone-sensitive (ER-positive) LABC, both anthracycline-/taxane-based chemotherapy and endocrine treatment alone are available as therapeutic options (LoE: IA). The decision must be made depending on the patient's individual situation (general condition, comorbidities, preference) and the tumor characteristics (grade, biomarker 
expression). The German experts add that the patient's menopausal status should also be considered in the therapy decision, and point out that endocrine treatment alone is reserved for individual situations, e.g., for clinically relevant comorbidity. The German experts emphasize that the use of chemotherapy is usually recommended in view of the still potentially curative setting.

\section{Special Metastatic Breast Cancer Settings}

\section{Focus on Liver Metastases}

The question cannot be answered whether and when patients with liver metastases will benefit from local treatment. The majority of the $\mathrm{ABC} 2$ panelists agreed that prospective clinical trials are urgently needed, since evidence-based data have up to now been available only for selected individual patient groups. Therefore, according to the consensus recommendation of the $\mathrm{ABC} 2$ panelists, the therapeutic decision must be discussed in an interdisciplinary team (tumor board). If there is the possibility to participate in a clinical trial, this should be preferred.

Therefore, local therapies are currently options for individual cases only. Candidates should have a good performance status, only limited metastasis in the liver, and no or well-controlled extrahepatic lesions. In the absence of relevant data, it is currently unclear which local treatment is the most appropriate one for each individual patient (LoE: expert opinion).

The German experts agree with the statement of the ABC2 panelists, emphasize the importance of an interdisciplinary board and underline that the local approach must remain reserved for individual patients who are in a stable disease situation under systemic treatment. Nonsurgical, locally ablative approaches such as laser-induced thermotherapy (LITT), selective internal radiation therapy (SIRT), transarterial chemoembolization (TACE), or radiofrequency ablation (RFA) may also be considered in individual cases.

\section{Focus on Malignant Pleural Effusion}

The majority of the ABC2 panelists recommend systemic and local treatment for patients with malignant pleural effusion. Despite a relatively high rate of false-negative results, they advocate thoracocentesis for diagnostic purposes. They recommend drainage for symptomatic patients with clinically relevant (moderate to massive) pleural effusion. In addition, talcum or cytostatic drugs, e.g. bleomycin, may be helpful for pleurodesis (LoE: II B).

The German experts advocate a palliative thoracocentesis only for symptomatic patients. As long as the patient's disease is stable, local treatment of a malignant pleural effusion for palliative reasons may be sufficient initially. In this case, a change of systemic therapy is not necessary. The German experts consider talc instillation (with video-assisted thoracoscopy (VATS)) for pleurodesis as the most effective palliative treatment option for patients with pleural effusion at this time and also recommend a cytological confirmation, if possible with determination of biomarkers (ER, PR, HER2). However, there are also reports in the current literature on continuous drainages in the outpatient setting.

\section{Chest Wall and Locoregional Recurrence}

Patients with a chest wall and/or locoregional recurrence (with involvement of lymph nodes) have a high risk of distant metastasis. The ABC2 panelists therefore recommend complete restaging with an evaluation of the lung, abdomen, and bones (LoE: expert opinion). The German experts agree with the panelists but add that, similar to the situation during the initial diagnosis, the medical history plus a clinical examination must precede the staging and that conventional imaging (chest X-ray, upper-abdomen sonography or CT, as well as bone scans) must be the initial focus (see above).

There was agreement that patients with a chest wall and/or locoregional recurrence should undergo surgery if this approach is feasible with low morbidity. In addition, the patients require locoregional radiation treatment if they were not previously irradiated (LoE: IB). However, in individual cases, repeated radiation of the chest wall or certain regions may also be considered (LoE: expert opinion).

The German experts point out that a $\mathrm{R} 0$ resection should be the goal of the surgical intervention. Thus, if required, extensive resection with subsequent repair using autologous tissue should also be considered. In this context, the German experts note that the tumor volume can also be reduced preoperatively by using preoperative systemic treatment. If a reradiation is performed, concomitant local hyperthermia can induce an improved local control. German centers offering this procedure are listed on the homepage of the German Cancer Society (DKG).

The vast majority of the $\mathrm{ABC} 2$ panelists recommended that patients with a chest wall and/or locoregional recurrence should also receive 'pseudo-adjuvant' systemic treatment following radical surgery and/or radiation (LoE: IB). Systemic treatment may consist of chemotherapy, endocrine treatment and/or anti-HER2 therapy. The choice of systemic treatment depends on different factors, like receptor status, pretreatment, and duration of the disease-free interval. 'Secondary adjuvant' chemotherapy has the best results in patients with an ER-negative recurrence.

The German experts note that the term 'pseudo-adjuvant' is not routinely used in Germany and prefer the term 'secondary adjuvant' systemic therapy. They also note that chemotherapy can be used preoperatively.

The ABC2 panelists and the German experts agreed that palliative systemic therapy based on therapeutic principles for the metastatic setting is appropriate for those patients for whom radical local therapy is not an option or is declined. 


\section{Updates Regarding Metastatic Breast Cancer}

\section{Focus on the Patient}

It is indisputable that all patients with advanced breast cancer must be fully informed about their disease and the treatment options. This must not only be done in an understandable way and reflect current scientific knowledge, but should also be realized with empathy and in consideration of the cultural background. The German experts add that the therapy decision will be made together with the informed patient ('shared decision-making').

The majority of the $\mathrm{ABC} 2$ panelists recommended to survey patients routinely with validated questionnaires regarding tumor-related complaints and therapy-related adverse events. This should be a regular component of the clinical management. The $\mathrm{ABC} 2$ panelists view such systematic monitoring as the basis for

- facilitating communication between patient and treatment team,

- guaranteeing the best-possible quality of life for the patient,

- better recognizing and characterizing toxicities of treatment. The last item is particularly important for newly approved drugs/therapies, especially for those whose approval is based only on an advantage in progression-free survival or on a moderate survival advantage (LoE: IC).

The German experts point out that regular recording of adverse events is part of standard oncological care in Germany and that adverse events are documented in the medical records. Recording using validated questionnaires and subsequent evaluation on a routine basis is desirable in principle but not feasible in clinical daily routine. The requirement to work routinely with validated questionnaires in daily clinical practice is therefore unrealistic and remains reserved primarily for patients in clinical trials.

There is agreement/consensus among the ABC panelists and the German experts that the patient's age in and of itself should not be the decisive factor for the choice of therapy decision. On the one hand, older patients should not be excluded from effective standard therapy just because of their age. On the other hand, aggressive treatment should not be triggered solely by young age (risk of overtreatment) (LoE: IB).

\section{Integration of Specialized Nurses}

The German experts agree with the recommendation of the $\mathrm{ABC} 2$ panelists that specialized oncology nurses, if possible specialized breast care nurses, should be part of the interdisciplinary treatment team (LoE: expert opinion). In Germany, specialized breast care nurses are mandatory team members in all certified breast centers.

\section{ER-Positive/HER2-Negative Metastatic Breast Cancer}

A clear majority of the $\mathrm{ABC} 2$ panelists agree that the combination of everolimus plus aromatase inhibitors can be a valid therapy option for patients with hormone receptor-positive metastatic breast cancer and endocrine resistance. The addition of everolimus to exemestane in the pivotal BOLERO-2 trial [2] resulted in a clinically meaningful extension of the median progression-free survival by approximately 5 months. Overall survival data are not yet available; therefore, the therapy decision should also take into account the increased toxicity associated with everolimus (LoE: IB). In addition, it should be noted that there are still no predictive biomarkers to identify those patients who benefit most from this approach.

The German experts basically agree with this statement. They also point out that the indication for adding everolimus to the aromatase inhibitor should be in line with the inclusion criteria of the BOLERO-2 trial [2]. The German experts emphasize that it is necessary to balance treatment benefits with the potentially increased toxicity compared to endocrine therapy alone.

\section{HER2 Blockade in HER2-Positive}

\section{Metastatic Breast Cancer}

For patients with HER2-positive metastatic breast cancer, the combination of chemotherapy plus trastuzumab is superior to the combination of chemotherapy plus lapatinib with respect to progression-free and overall survival. This applies regardless of whether patients are already pretreated with trastuzumab or not (LoE: IA). The majority of the ABC2 panelists agree with this statement. The German experts confirm that this statement is consistent with current data and point out that new evidence has been provided by the MA.31 [3] and the CEREBEL studies since ABC1 [4].

The majority of the $\mathrm{ABC} 2$ panelists recommend dual HER2 blockade with trastuzumab plus pertuzumab (in addition to taxane chemotherapy) as the preferred therapy option for first-line treatment (LoE: 1A), especially for patients without any anti-HER2 pretreatment. Current data from the CLEOPATRA study [5, 6] show that dual HER2 blockade with pertuzumab plus trastuzumab plus docetaxel is superior to the combination of trastuzumab plus docetaxel with regard to progression-free and overall survival. The German experts agree since this recommendation is in line with the updated German Gynecological Oncology Working Group (Arbeitsgemeinschaft gynäkologische Onkologie (AGO)) guidelines (www.ago-online.de). From the German perspective, dual HER2 blockade with pertuzumab plus trastuzumab together with docetaxel chemotherapy is a new standard for first-line therapy of HER2-positive metastatic breast cancer.

The ABC2 panelists and the German experts also agree on the following items: At this time it is still unclear how the new dual HER2 blockade performs compared to other antiHER2-based therapy options, e.g., compared to the antibodydrug conjugate trastuzumab emtansine (T-DM1). There are currently no data to support routine use of dual HER2 blockade with pertuzumab plus trastuzumab in later therapy lines
56

Breast Care 2014;9:52-59
Harbeck/Marschner/Untch/Decker/HegewischBecker/Jackisch/Huober/Lück/von Minckwitz/ Scharl/Schneeweiss/Tesch/Welt/Wuerstlein/ 
(i.e. after first-line treatment). The German experts add that there are also no data on the repeated use of dual HER2 blockade with the same anti-HER2 agents across multiple lines, i.e. treatment beyond progression (TBP).

The ABC2 panelists evaluated the antibody-drug conjugate T-DM1 after failure of the trastuzumab-containing firstline therapy as the most effective option for the subsequent therapy lines (LoE: IA) [7,8]. The German experts agree with this statement.

According to the ABC2 panelists, it is basically unclear at this time which therapy option is the best one for metastatic patients with HER2-positive breast cancer and recurrence following adjuvant anti-HER2-containing treatment. Yet, it is undisputed that patients require another line of anti-HER2based treatment (LoE: IB). Finally, the ABC2 panelists state that the choice of the anti-HER2-based regimen depends on the circumstances in the particular country and the available drugs, as well as on the anti-HER2-based pretreatment and the length of the recurrence-free interval. The optimal sequence of available anti-HER2-based therapies is still unclear at the moment [9].

The German experts recommend that patients who do not have a recurrence or metastasis for more than 1 year after the end of adjuvant trastuzumab administration can receive firstline treatment with pertuzumab plus trastuzumab plus docetaxel, in accordance with the CLEOPATRA study [5, 6]. The optimal further treatment for patients with early metastasis or only a short recurrence-free period $(<1$ year after the end of adjuvant trastuzumab) is unclear. From the German perspective, the following options are possible: T-DM1, capecitabine plus lapatinib, taxane plus trastuzumab plus pertuzumab, and trastuzumab plus lapatinib. With regard to the optimal sequence, the German experts refer to the respective approval status. They also point out that $\mathrm{ABC} 1$ recommended that in patients with ER+ and HER2+ metastatic breast cancer for whom endocrine therapy was chosen over chemotherapy, anti-HER 2 plus endocrine therapy should be considered with the initiation of endocrine therapy (provided that further anti-HER2 therapy is available) since anti-HER2 therapy (either trastuzumab or lapatinib) in combination with endocrine therapy has shown substantial progression-free survival benefit (i.e. 'time without CT') even though no survival benefit compared to endocrine therapy alone (LoE: $1 \mathrm{~A})$. This approach may be an option for patients with oligo-metastatic disease or comorbidities.

\section{Brain Metastases in Patients with}

\section{HER2-Positive Breast Cancer}

The survival time of patients with HER2-positive breast cancer and brain metastases has improved in recent years. A clear majority of the $\mathrm{ABC} 2$ panelists therefore recommended to pay particular attention to the toxicity of treatment and to use (if possible) less toxic local treatments, e.g., stereotactic radiation.

The German experts note that the therapeutic standards for the treatment of brain metastases apply regardless of the HER2 status and should be followed. In addition to neurosurgical treatments and total brain radiation, stereotactic radiation can also be an option. In general, the German experts recommend total brain radiation.

\section{HER2-Negative Metastatic Breast Cancer}

A majority of the $\mathrm{ABC} 2$ panelists recommended that the preferred endocrine first-line treatment for postmenopausal patients with HER2-negative and ER-positive metastatic breast cancer is either an aromatase inhibitor or tamoxifen. The therapy decision depends on the type and duration of the adjuvant endocrine pretreatment (LoE: IA). High doses of fulvestrant are an alternative (LoE: IB). The German experts refer to the current $\mathrm{AGO}$ guidelines (www.ago-online.de) for the therapy decisions and confirm that the optimal individual first-line endocrine therapy is based on the duration and type of pretreatment (tables 2 and 3 ).

For patients with HER2-negative metastatic breast cancer who have been pretreated with anthracyclines and taxanes and do not require combination chemotherapy, the $\mathrm{ABC} 2$ panelists consider cytostatic monotherapy with capecitabine, vinorelbine, carboplatin, or eribulin as the preferred therapy

\begin{tabular}{llll}
\hline Treatment sequence & \multicolumn{2}{l}{ Oxford LoE / AGO GR } \\
\hline First line & aromatase inhibitors (3rd generation) $)^{\mathrm{a}}$ & 1a A & ++ \\
& fulvestrant $250 \mathrm{mg}+$ anastrozole & $1 \mathrm{~b} \mathrm{~B}$ & \pm \\
Second line & fulvestrant & $2 \mathrm{~b} \mathrm{~B}$ & \\
& $\quad$ fulvestrant $500 \mathrm{mg}$ & $2 \mathrm{~b} \mathrm{~B}$ & ++ \\
& $\quad$ fulvestrant $250 \mathrm{mg}$ & $1 \mathrm{~b} \mathrm{~B}$ & + \\
& exemestane + everolimus & $1 \mathrm{~b} \mathrm{~B}$ & ++ \\
& tamoxifen & $3 \mathrm{~b} \mathrm{C}$ & + \\
& aromatase inhibitors & $2 \mathrm{~b} \mathrm{~B}$ & + \\
Other therapy lines & tamoxifen + everolimus & $2 \mathrm{~b} \mathrm{~B}$ & + \\
& MPA / MA & $4 \mathrm{D}$ & + \\
& estradiol 6 mg daily & $3 \mathrm{~b} \mathrm{C}$ & + \\
& reinduction of previous therapies & $5 \mathrm{D}$ & + \\
\hline
\end{tabular}

Table 2. Endocrine therapy of postmenopausal, hormone receptor-positive, HER2negative metastatic breast cancer following adjuvant tamoxifen therapy or without adjuvant endocrine therapy (modified from www.ago-online.de)

\footnotetext{
${ }^{\text {a }}$ There is no evidence of the superiority of an individual aromatase inhibitor.

bSteroidal or non-steroidal aromatase inhibitor, depending on the previous one.

$\mathrm{GR}=$ Grade of recommendation, $\mathrm{MPA}=$ medroxyprogesterone acetate, $\mathrm{MA}=$ megestrol acetate .
} 
Table 3. Endocrine therapy of postmenopausal, hormone receptor-positive, HER2-negative metastatic breast cancer following adjuvant therapy with an aromatase inhibitor (modified from $w w w$. ago-online.de)

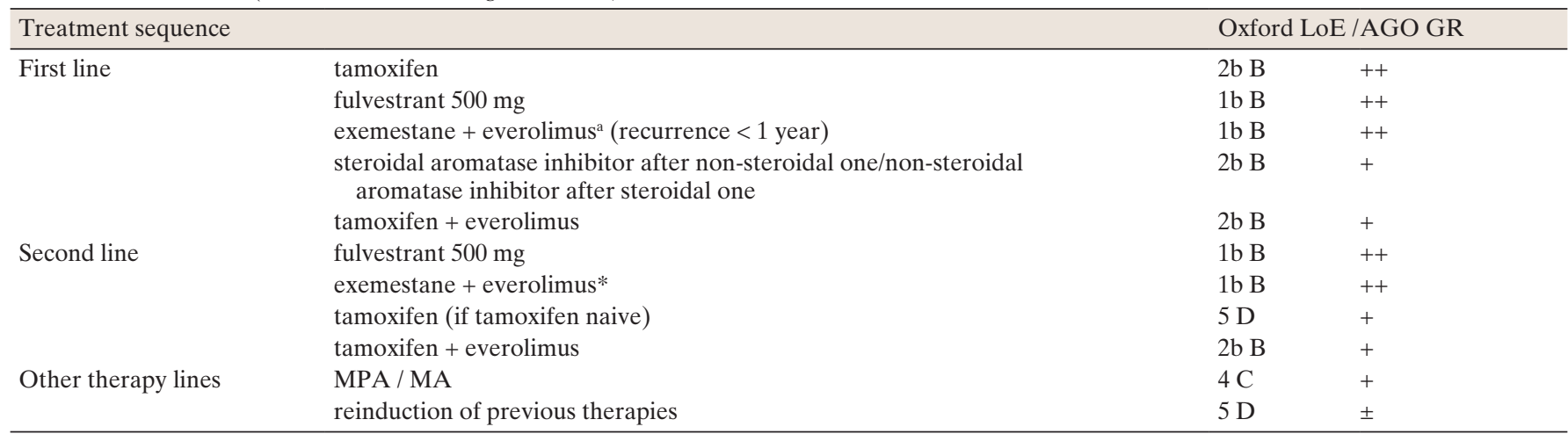

${ }^{a}$ After pretreatment with at least one non-steroidal aromatase inhibitor (metastatic and/or adjuvant).

$\mathrm{GR}=$ Grade of recommendation, $\mathrm{MPA}=$ medroxyprogestrone acetate, $\mathrm{MA}=$ megestrol acetate.

option. When choosing a therapy, the differences in the side effect profile of the drugs should be considered (LoE: IB). The German experts agree and add that repeated administration (re-challenge) of a taxane (e.g., docetaxel, nab-paclitaxel, or paclitaxel - whatever had not been given in the early breast cancer setting) or an anthracycline such as (peg-)liposomal doxorubicin are also valid options for cytostatic monotherapy. However, they also point out that there is not sufficiently validated data to support monotherapy with gemcitabine in metastatic breast cancer.

\section{Male Breast Cancer}

Male breast cancers are generally hormone sensitive (positive hormone receptor status). Therefore, endocrine therapeutic treatments, preferably with tamoxifen, are the therapy of choice. If an aromatase inhibitor is used, it should be given with a luteinizing hormone-releasing hormone (LHRH) agonist; an alternative to the LHRH agonist is an orchiectomy. Exclusive aromatase inhibitor therapy is an option if the patient's response to therapy is closely monitored. The ABC2 panelists and the German experts agree on these points and note the urgent need for clinical trials for men with breast cancer (such as the ongoing GBG MALE trial, www.germanbreastgroup.de) to answer this as well as other open questions.

\section{Focus on Endocrine Resistance}

There was an intense debate in Lisbon on primary and secondary endocrine resistance of the tumor and how to define them. The German experts do not agree with the definitions of the $\mathrm{ABC} 2$ Consensus, where primary endocrine resistance for early breast cancer is defined as recurrence within the first 2 years of adjuvant endocrine therapy and, for metastatic breast cancer, as progression within 6 months after the start of ongoing first-line endocrine treatment. The majority of the ABC2 panelists define acquired (secondary) endocrine resistance for early breast cancer as recurrence during adjuvant endocrine therapy after more than 2 years or within 1 year after the end of adjuvant endocrine treatment. In the opinion of the $\mathrm{ABC} 2$ panelists, secondary endocrine resistance in metastatic breast cancer is assumed if there is progression only after more than 6 months after start of the current endocrine treatment.

The German experts note that endocrine resistance develops continuously; therefore, an arbitrary differentiation between primary and secondary resistance is clinically difficult. The German experts emphasize that starting a different endocrine therapy after endocrine failure generally makes sense in daily clinical practice, regardless of the time of progression in case of a positive hormone receptor status. Chemotherapy is primarily indicated only for rapidly progressing disease with symptoms that require a fast therapy response.

\section{ABC Patient Advocacy Committee}

At this years' ABC meeting, breast cancer advocacy organizations $(n=49)$ met separately from the scientific session, and a report from the ABC Patient Advocacy Committee was presented during the plenary consensus. The committee emphasized the need for multidisciplinary team (MDT) discussions using ABC-specific guidelines, patient navigators, continuity of oncologists and nurses in patient care, improved communication between the patients and the MDT, as well as knowledge and consideration of the patients' priorities. Other issues to be considered include supportive care for the patients themselves, their families and caregivers, and nutrition, sexual dysfunction, complementary medicine, and rehabilitation. The different national healthcare systems need to enable easy access to clinical trials, standardized and affordable treat- 
ment standards, compliance monitoring, and development of tumor banks and patient registries. Last but not least, further economic and social needs were mentioned such as maintaining employment, disability coverage, stigmatization due to $\mathrm{ABC}$, and the need for support regarding cancer- or therapyrelated lifestyle changes. Since patient advocates are also part of the ABC consensus panel, all of these issues will be considered upon finalization of the official $\mathrm{ABC}$ slide set and manuscript.

Altogether, the ABC2 was a very informative discussion of recent developments in breast cancer. The $\mathrm{ABC}$ consensus is therefore a good addition to the St. Gallen Conference. ABC3 is already scheduled for November 5-7, 2015. In Germany, the updated AGO guidelines will be presented in Frankfurt on March 1, 2014.

\section{Disclosure Statement}

The Meeting of the German Experts in Lisbon was supported by Celgene GmbH. The manuscript was drafted by Birgit-Kristin Pohlmann, Nordkirchen. Final approval of the manuscript rested solely with the authors.

\section{References}

1 Buzdar A, et al.: ACOSOG Z1041 Study. J Clin Oncol 2013;31(suppl):abstr 502.

2 Baselga $\mathrm{J}$, et al.: Everolimus in postmenopausal hormone-receptor-positive advanced breast cancer. N Engl J Med 2012;366:520-529.

3 Gelmon KA, et al.: MA.31 Study, Proc ASCO 2012. J Clin Oncol 2012;30(suppl):abstr LBA671.

4 Pivot J, et al.: CEREBEL Study. Ann Oncol 2012; 23(suppl 9):IXe1-IXe30.
Baselga J, et al.: Pertuzumab plus trastuzumab plus docetaxel for metastatic breast cancer. N Engl J Med 2012;366:109-119.

6 Swain S, et al.: Confirmatory overall survival (OS) analysis of CLEOPATRA: a randomized, doubleblind, placebo-controlled phase III study with pertuzumab (P), trastuzumab (T), and docetaxel (D) in patients (pts) with HER2-positive first-line (1L) metastatic breast cancer (MBC). Cancer Res 2012;72 (24 suppl):476s
7 Verma S, et al: Trastuzumab emtansine for HER2positive advanced breast cancer. N Engl J Med 2012;367:1783-1791.

8 Wildiers $\mathrm{H}$, et al.: T-DM1 for HER2-positive MBC: primary results TH3RESA, a phase 3 study of T-DM1 vs treatment of physician's choice. Proc ECC 2013; abstr LBA 15.

9 Harbeck N, Wuerstlein R: Optimal sequencing of anti-HER2 therapy throughout the continuum of HER2-positive breast cancer: evidence and clinical considerations. Drugs 2013;73:1665-1680. 